\title{
COMPARISON BETWEEN POINT-BASED AND RING-BASED TECHNOLOGIES IN KERATOMETRIC MEASUREMENT AMONG POST MYOPIC LASER REFRACTIVE SURGERY PATIENTS
}

\author{
Md Muziman Syah ${ }^{1}$, Aminul Hakim Sofian Sauri ${ }^{1}$, Khairidzan Mohd Kamal ${ }^{2}$, Mahfuzah \\ Mokhtar ${ }^{1}$
}

${ }^{1}$ Department of Ophthalmology, Kulliyyah of Medicine, International Islamic University, Pahang, Malaysia, ${ }^{2}$ Department of Optometry and Visual Science, Kulliyyah of Allied Health Sciences, International Islamic University, Pahang, Malaysia.

Presenter: Aminul Hakim Sofian Sauri, aminulhakim.optom91@gmail.com

Introduction: Corneal power or keratometric value represents two-third of total refractive power of the eye. It is an important parameter in intraocular lens calculation. Purpose of the study was to evaluate repeatability and agreement of keratometric measurements obtained from a six points-based keratometry device; IOLMaster 500 (Carl Zeiss Meditec AG, Jena, Germany) and two Placido disc-based keratometry devices; Atlas Topographer 995 and 9000 models (Carl Zeiss Meditec AG, Jena, Germany).

Materials and method: A total of 97 post myopic laser refractive surgery subjects were recruited. Three set of mean corneal power measurements from each device were performed by an examiner in a single visit. The repeatability was evaluated by withinsubject standard deviation, coefficient of variation and intraclass correlation coefficient. A one-way repeated measures analysis of variance was conducted to evaluate keratometric measurement differences between the devices. The BlandAltman analysis was employed to assess inter-device agreement.

Results: There were no significant differences of three repeated keratometric measurements in each device $(p>0.05)$. IOLMaster showed the lowest coefficient of variations in all meridians $(<0.30 \%)$. The intraclass correlations were higher than 0.90 in all devices. For inter-device agreement, there were statistically significant differences between Atlas 9000-IOLMaster and Atlas 995-IOLMaster $(p<0.05)$. The $95 \%$ limit of agreement ranged from 0.94 to 1.68 .

Conclusion: The six points-based technology has a higher repeatability compared to ring based technology in measuring postoperative keratometric measurements. Different keratometry technologies cannot be used interchangeably in clinical setting. 